\title{
Fixing necrotizing pancreatitis
}

A new study published in Pancreas shows that the anti-inflammatory effects of central $\alpha 2$ agonists, which are already in clinical use, are able to decrease the severity of experimental necrotizing pancreatitis in rats.

Necrotizing pancreatitis is a complication of acute pancreatitis, with high morbidity and mortality. At present, endoscopic approaches are the main method for treating this condition.

The anti-inflammatory effects of vagal nerve stimulation have been suggested to ameliorating necrotizing pancreatitis in experimental studies. The central $\alpha 2$ agonists clonidine and dexmedetomidine are used clinically for their sedative properties and are known to increase parasympathetic tone, including in the vagus nerve. The authors used these compounds to see if pancreatic injury could be improved in a rat model of severe necrotizing pancreatitis.

Disease was induced in rats by infusion of glycodeoxycholic acid into the pancreatic duct. Rats were given either clonidine or dexmedetomidine in a prophylactic or therapeutic fashion before samples were harvested. Control animals were treated with saline.

Prophylactic treatment with either compound reduced oedema, inflammation and necrosis compared with rats given saline. Pancreatic necrosis was substantially reduced by therapeutic clonidine or dexmedetomidine treatment compared with control animals.

Both compounds and treatment methods also resulted in a decrease in serum levels of high mobility group protein $\mathrm{B} 1$, a proinflammatory cytokine involved in systemic inflammatory damage associated with pancreatitis.

The authors write that in the future, “... a clincal trial with the usage of central $\alpha 2$ agonists as adjunct sedative medication with focus on post-ERCP pancreatitis seems to be an applicable idea ..."

Gillian Patman

ORIGINAL ARTICLE Schneider, L. et al. Stimulation of central $\alpha 2$ receptors attenuates experimental necrotizing pancreatitis. Pancreas 45, 260-264 (2016) 